\title{
Compact, Lightweight, Superconducting Power Generators
}

\author{
Paul N. Barnes, Gregory L. Rhoads, Justin C. Tolliver, Michael D. Sumption, Kevin W. Schmaeman \\ Air Force Research Laboratory \\ AFRL/PRPG \\ Wright-Patterson AFB, OH 45433 \\ paul.barnes@wpafb.af.mil
}

\begin{abstract}
Many future military systems will depend heavily on high electrical power input ranging from 100's kilowatts up to the multimegawatt level. These weapon systems include electromagnetic launch applications as well as electrically driven directed energy weapons (DEW), such as high power microwaves and solid state lasers. These power generation subsystems must often be packaged using limited space and strict weight limits on either ground mobile or airborne platforms. Superconducting generators made of high temperature superconductors (HTS) will enable megawatt-class airborne power systems that are lightweight and compact. Also discussed briefly are new advances in HTS conductors and refrigeration systems furthering the development of HTS power systems.
\end{abstract}

Keywords-generators; high electrical power; superconducting; HTS conductors

\section{INTRODUCTION}

Future revolutionary weapon systems in the military will depend heavily on high electrical power input at the multimegawatt level. These weapon systems include electrically driven directed energy weapons (DEW), such as high power microwaves and solid state lasers, as well as electromagnetic rail launch. Not only do these innovative weapon systems require significant amounts of reliable electrical power, they often must be packaged in a limited space and within strict weight limits on either ground-mobile or airborne platforms. As such, space and weight are at a premium for these systems. In the Air Force, a new class of small, lightweight, \& high power generators is being developed using superconducting technology. Superconducting generators made of high temperature superconductors (HTS) will enable megawatt-class airborne power systems. This paper will examine the current state of superconducting generator work in the Air Force and, in particular, its relation to airborne DEW. Also discussed briefly are new advances in flux pinning and ac loss reduction for HTS conductors will further the development of HTS generator systems.

Conventional generators that provide high electrical power have been developed and optimized over the past several decades, but these generators cannot provide the multimegawatt level of power required for advanced mobile or airborne military systems without paying a significant penalty in size and weight. Efficiency, thermal management, and

Work supported by the Air Force Research Laboratory fatigue life are typically all sacrificed as conventional generators are given high rotational speeds to reduce their size and weight while maintaining high power output. To circumvent these issues, superconducting generators are being developed that maintain high efficiencies, have lower thermal management requirements, and are lightweight and compact.

An earnest effort at developing high power superconducting generators for airborne applications began in the 1970's with an alternator development program that used a low temperature superconductor (LTS), NbTi, for the rotor windings [1]. One issue with this initial development program is the necessity to cryogenically cool the rotor windings down to $4.2 \mathrm{~K}$. Even so, the electromagnetic shield on the rotor was considered the most significant problem of the superconducting alternator suggesting that future designs have separate thermal and electromagnetic shields to avoid excessive heating in the shield due to load-induced varying fields. The combination of poor cooling and high losses placed strict design constraints on the superconductor specifications.

These concerns led to a subsequent $40 \mathrm{MW}$ generator development incorporating high-purity "hyperconducting" aluminum in the windings. This composite aluminum conductor is not superconducting, but has an extremely low electrical resistance at liquid hydrogen temperatures, $\sim 20 \mathrm{~K}$ [2]. A $600 \mathrm{~kW}$ exciter, also using the composite aluminum, constructed for the $40 \mathrm{MW}$ generator weighed approximately $100 \mathrm{~kg}$ [3]. Based on the design and data collected, it was speculated that the exciter could have provided $1 \mathrm{MW}$ of power, but testing was not done at this level. A main drawback of the composite aluminum conductor was the use of liquid hydrogen due to its limited availability in most applications. As such, "ceramic superconductors will generally be more practical to cool in similar applications when they achieve the necessary development status [2]." The first HTS ceramic superconductor to be made in practical lengths was bismuth strontium calcium copper oxide (BSCCO).

With the development of the BSCCO wire a new program was initiated to incorporate the bismuth-based superconductor into the previously constructed $600 \mathrm{~kW}$ exciter by replacing the composite aluminum field windings with HTS BSCCO field windings [4]. Eight field winding poles were fabricated, tested, and met the required specification (72,000 Amp-turn). However, after the windings were delivered to the prime 
contractor to retrofit the exciter, two of the coils were destroyed during a welding process by a sub-contractor on the program. Additional funds were not available to procure the replacement coils for final testing of the generator and testing never occurred. The use of BSCCO can raise the operating temperature to $20-30 \mathrm{~K}$.

Two major improvements have occurred since this time. One is the development of the second generation HTS conductor, $\mathrm{YBa}_{2} \mathrm{Cu}_{3} \mathrm{O}_{7-\mathrm{x}}$ or $\mathrm{YBCO}$, which can operate in the temperature range of 60-77 K. A second important advance is the steady improvement in cryocoolers and refrigeration systems that have greatly reduced their size and improved their efficiency. The improvements offered by YBCO, discussed later in this paper, make it a critical material for future defense applications which require high electrical power. These systems include power components for directed energy weapons (DEW) such as high power microwaves, electrically driven lasers, electromagnetic railguns, electric ship concepts such as electric propulsion, electromagnetic launch, etc. Potential components for these systems that can use the HTS conductor include gyrotron magnets, power generators, power converters (inductors), transformers, motors, primary power cabling, and MHD magnets.

HTS conductor availability is promoted by a variety of commercial applications. The primary commercial demand for the coated conductor will be electric power equipment. Complete development of the technology will lead to industrial commercialization of the HTS coated conductor in electric power applications such as transformers, transmission cables, motors, fault current limiters, synchronous condensers and generators. The overall advantages of using HTS conductor are a significant reduction in size and weight with the associated increase in power density, significant reduction in energy usage due to low electrical losses, and noise reduction in HTS machinery and transformers. Specific advantages include: HTS cable with 3-5 times more power capacity than conventional, elimination of fire and environmental hazards of cooling oil in transformers, a 4-6 fold reduction in fault current levels with HTS fault current limiters with no capacitive correction, reduced production cost for HTS motors which are more electrically stable during transients, and HTS generators needing less spinning reserve for stability and having overexcitation capability without added synchronous reactance.

A common misconception regarding the use of the superconductors is the need for large amounts of liquid cryogens to make the application functional. This misconception comes from earlier development activities when LTS materials were used and before the recent improvements in cryocoolers. YBCO can operate at liquid nitrogen temperatures which can readily be achieved by compact durable mechanical refrigeration systems which use no liquid cryogens. An HTS ship propulsion motor made with BSCCO conductor, operating at $\sim 30 \mathrm{~K}$, was one-third the size and half the weight of its conventional counterpart and this includes the cooling system [5]. No extra effort was taken to make it lighter or more compact.

There is a tendency for designers of conventional high power airborne generators to ignore the burden of thermal management. Airborne conventional generators are significantly less efficient. For example, if $5 \mathrm{MW}$ of power is produced at $85 \%$ efficiency, then $750 \mathrm{~kW}$ of heat must be dumped somewhere from the aircraft. At low overall powers, this figure is significantly less and does not pose as great a problem. Superconducting generators can maintain high efficiencies at lighter weights.

\section{MILITARY APPLICATIONS}

Some of the past Air Force development activities, such as the 40MW Westinghouse generator, were specifically in support of electromagnetic launch (EML) applications. For EML, the Air Force had concentrated on homopolar generators and built and tested a superconducting LTS version with General Atomics at 550 Volts. The pulse requirements for EML generated huge ac losses and the cryogenic aluminum work was specifically developed to focus on solving this problem. Also, the large current switching issues have also been a problem area of the past which may be addressed by HTS materials [6]. Technologies such as EML and electromagnetic railguns require pulsed power, but what is often overlooked is the fact that the required average power of these application may extend into the megawatt range and may require a continuous electrical power generation to charge the energy storage system, whether capacitor banks, pulse forming networks, etc. [7] Further, the superconducting generator has important advantages in specific weight and applications requiring a high voltage output at high power.

Another application of great interest is directed energy weapons. Airborne active denial technology (AADT) represents a paradigm shift in airborne weaponry providing non-lethal anti-personnel capabilities via high power microwaves. This system will offer field commanders a nonlethal option, enabling the relaxation of the rules of engagement to allow first use of non-lethal force. The Air Force is initiating new superconducting generator development activities that take advantage of the recent developments in HTS materials and cryocoolers as well as the success of the last programs to address the high power requirements of this system. Further, the AADT requires a gyrotron magnet for the AADT source tube which currently uses low temperature superconductors (LTS). Replacing the current LTS conductors with HTS YBCO coated conductor allows cooling at $77 \mathrm{~K}$ as opposed to $4 \mathrm{~K}$ with an up to 100 -fold reduction in cooling requirements.

\section{SUPERCONDUCTING GENERATORS}

Basic synchronous superconducting generators can be subclassified into two primary types. The first is designated as the classical superconducting generator. This is the primary design that has been used to date and uses the superconductor only in the rotor windings. The stator is made of a conventional material, typically copper. In the rotor windings, the superconductor will primarily experience a dc field and as such ac losses are low. Efficiencies of the structure are high since the superconductor has zero resistance and hence no ohmic losses with dc current. However, there are issues associated with isolating the rotating cryogenic vessel from the room temperature stator and cryogenic connections to the spinning 
rotor. The high magnetic fields produced by the coil windings eliminate the need for iron to concentrate the field as in conventional generators.

The other class of superconducting generator is the allcryogenic superconducting generator. In this case, both rotor and stator windings are made of superconducting material allowing the entire generator to reside in a cryogenic jacket and cooled to the operating temperature. The armature windings will experience significant fluctuating magnetic fields inducing large ac losses. These ac losses can include hysteretic losses in the superconductor, normal metal effects and ferromagnetic contributions from the substrate of the HTS conductor, and coupling eddy currents. To successfully incorporate HTS conductor into the stator will require additional development of an ac tolerant YBCO coated conductor. Until such time, classical superconducting generators will be the workhorse for superconducting power systems.

\section{A. Military Advantages}

High temperature superconducting generators and magnets are significantly lighter and more compact than their conventional counterparts and the development of these devices are essential to applications that require compact, lightweight, high power sources or compact high field magnets, especially ground mobile, airborne, and naval applications. The superconducting generator has many advantages over conventional generators that have been mentioned previously. However, another major advantage of the HTS generator is the elimination of the iron needed in a conventional design. The magnetic fields that are produced by the superconductor are high enough that no iron is needed to direct the field lines. This leads to an "air core" design for the HTS generator. Elimination of the iron alone will greatly reduce weight. Combine that with weight savings of using a material with a much higher current density and the advantage is enormous - a total of an $80-90 \%$ reduction in weight of the generator compared to a typical commercial design.

High speed HTS generators especially offer great promise for producing multimegawatts of electrical power at voltages up to several thousand volts in a generator that potentially weighs a fraction of the weight of a traditional iron-based generator. A classic superconducting generator-HTS rotor only-using the YBCO coated conductor can conservatively achieve $0.1 \mathrm{~kg} / \mathrm{kW}$ power density. An all cryogenic generator can push this to a power density of $0.05 \mathrm{~kg} / \mathrm{kW}$. Iron-core high speed aircraft generators typically produce only 10's to at most a few hundred kilowatts at voltages generally $270 \mathrm{Vdc}$ or below. Extending their rating to several megawatts at thousands of volts presents serious design issues and proposed power densities tend to be very aggressive. Iron-core low speed commercial generators are available with the proper ratings, but are too heavy for multi-megawatt airborne or ground mobile applications.

\section{B. Commercial Advantages}

Commercially, the primary application of superconducting generators will be utility generation facilities, using either new or retrofitted generators. Benefits of commercial HTS generators include increased machine efficiency above $99 \%$ reducing losses by half over conventional generators, energy savings, reduced pollution per unit of energy produced, lower life-cycle costs, enhanced grid stability, and reduced capital cost and installation expenses. By using HTS wire for the field windings, losses in the rotor windings can be mostly eliminated. Further, the fields created in the armature by the rotor are not limited by the saturation characteristics of iron. The armatures are constructed without iron teeth removing the losses experienced in the armature teeth. Since HTS generators can produce electric power with lower losses, a substantial energy savings can be realized.

A commercial HTS generator is expected to be $1 / 3$ the overall volume of its conventional equivalent. In power plants where expansion is difficult such as shipboard or locomotive power, superconducting generators can increase generating capacity without using additional space. Smaller, lighter HTS generators use an air core design, as previously mentioned, eliminating much of the structural and magnetic steel of the conventional counterpart. Construction, shipping, and installation are all simplified and less costly. Also, HTS generators have lowered armature reactance which can significantly impact utility stability considerations. One implication is a reduction in the amount of spinning reserve (unused but rotating generating capacity) needed to insure a stable overall power system [8]. Another benefit is that an HTS generator has the capability of being significantly overexcited to permit power factor correction without adding synchronous reactors or capacitors to the power system [8].

\section{Refrigerators}

The significantly higher operating temperatures of the HTS conductors over LTS conductors is a major breakthrough for using superconductors in military systems. The operating temperatures for BSCCO $(30 \mathrm{~K})$ and YBCO (77K) HTS conductors eliminate the need for liquid cryogens. Cryogenic refrigerators (cryocoolers) are able to cool the HTS conductors without the complications and logistics of a liquid cryogen system. Reliable cryocoolers have been commercially available for several decades but are too large and not adapted to military environments.

The workhorse of the cryocoolers is the Gifford-McMahon (G-M) regenerative cycle cryocooler. Besides not being very efficient, the G-M cryocoolers cannot tolerate the military environment. The compressors used in G-M cryocoolers cannot be subjected to shock and tilting situations. The Air Force has recently developed a replacement compressor that is oil free, robust and operates at any angle. The replacement compressor uses a linear motor to directly drive the pistons that have clearance seals to eliminate the need for oil.

Gifford-McMahon cryocoolers are slowly being replaced by more advanced efficient cryocoolers such as Stirling and Pulse Tube cryocoolers. Stirling cryocoolers have been used by the military for decades for sensor cooling but these cryocoolers are too small for cooling larger volume superconducting applications. Large Stirling and Pulse Tube cryocoolers have just become available commercially after more than a decade of development. One cryocooler, recently developed by the Air Force for a laser application, is a 
lightweight Stirling cryocooler weighing only $19 \mathrm{lbs}$ and delivering 16 watts of refrigeration at 80 degrees Kelvin [10].

Cryocoolers like this Stirling model will be very important for the utilization of YBCO superconductors in military systems. The Air Force is also developing large Pulse Tube cryocoolers that will provide enough cooling for an all cryogenic generator. This cryocooler is scheduled to be demonstrated in 2004 and will provide 300 watts of cooling at 65 degrees Kelvin. Both of these cryocoolers are using linear motor compressors that enable them to be lightweight, robust, reliable, attitude free and efficient military cryocoolers.

\section{YBCO COATED CONDUCTORS}

HTS conductors are typically formed in the shape of a thin tape that allows the conductor to be bent around relatively small diameters for high density coil windings. In the case of BSCCO, especially $\mathrm{Bi}_{2} \mathrm{Sr}_{2} \mathrm{Ca}_{2} \mathrm{Cu}_{3} \mathrm{O}_{\mathrm{x}}$, the thin tape is fabricated as a multi-filament composite superconductor that includes individual superconducting filaments the length of the conductor and are surrounded by a largely silver matrix. Alternatively, YBCO uses coated conductor technology as the method of fabrication. In this case, the superconductor exists as a thin coating on a flexible metallic substrate. Between the substrate and the superconductor are additional thin buffer layers for compatibility issues. The substrate is usually a metallic alloy and a final protective layer of silver is applied on top of the superconductor to serve as a protective layer. A copper stabilizing layer can be subsequently added as required.

YBCO is accepted world-wide as the future second generation HTS conductor. BSCCO is the present day, first generation HTS conductor. The primary advantages of YBCO superconductors include its use at liquid nitrogen temperatures $(77 \mathrm{~K}$ ) for reduced cooling requirements compared to $4 \mathrm{~K}$ for LTS and 20-30 K for BSCCO (due to poor in-field performance at $77 \mathrm{~K}$ ), ability to maintain current densities $>10^{5}$ $\mathrm{A} / \mathrm{cm}^{2}$ in Tesla level magnetic fields, good pinning properties and substantial reduction in ac losses over BSCCO conductors which are over $50 \%$ silver-also a costly material. Recently, the strain tolerance of the YBCO conductor was demonstrated to be superior to the BSCCO conductor, a critical factor for generator windings [11]. Table I summarizes the advantages.

TABLE I. HTS CONDUCTOR COMPARISON

\begin{tabular}{|l|c|c|l|}
\hline \multirow{2}{*}{ Property } & \multicolumn{3}{|c|}{ Comparison } \\
\cline { 2 - 4 } Cost & $\boldsymbol{B S C C O}$ & $\boldsymbol{Y B C O}$ & \multicolumn{1}{|c|}{ Advantage } \\
\hline $\begin{array}{l}\text { Operating } \\
\text { Temp. }\end{array}$ & $20-30 \mathrm{~K}$ & $60-77 \mathrm{~K}$ & $\begin{array}{l}\text { reduced cooling } \\
\text { requirements }\end{array}$ \\
\hline AC Losses & $\begin{array}{c}\text { Moderate to } \\
\text { heavy }\end{array}$ & $\begin{array}{c}\text { Low to } \\
\text { moderate }\end{array}$ & $\begin{array}{l}\text { Losses } \\
\text { minimized }\end{array}$ \\
\hline $\begin{array}{l}\text { Eng. } \\
\text { Current } \\
\text { Density }\end{array}$ & $12-15 \mathrm{kA} / \mathrm{cm}^{2}$ & $15-20 \mathrm{kA} / \mathrm{cm}^{2}$ & $\begin{array}{l}\text { Less wire for } \\
\text { equiv. amp-turns }\end{array}$ \\
\hline $\begin{array}{l}\text { Pinning } \\
\text { Strength }\end{array}$ & $<1 \mathrm{~A} / \mathrm{cm}^{2}$, & $>10^{5} \mathrm{~A} / \mathrm{cm}^{2}$, & $\begin{array}{l}\text { BSCCO already } \\
\text { enhanced, } \\
\text { YBCO can be }\end{array}$ \\
\hline $\begin{array}{l}\text { Critical } \\
\text { Strain }\end{array}$ & $\begin{array}{c}3 \mathrm{~T}, 77 \mathrm{~K} \\
\text { (marginal) }\end{array}$ & $\begin{array}{c}\text { Y.15 }-0.20 \% \\
\text { (good) }\end{array}$ & $\begin{array}{l}\text { BSCCO tensile } \\
\text { strain borderline }\end{array}$ \\
\hline
\end{tabular}

Advances in the YBCO coated conductors have resulted in dramatic improvements in the quality and length of wire available-from $10^{5} \mathrm{~A} / \mathrm{cm}^{2}$ at $\mathrm{cm}$ lengths about 3 years ago to lengths of several meters with $10^{6} \mathrm{~A} / \mathrm{cm}^{2}$ performance. These successes have been achieved by improved deposition methods for the HTS layer, more robust and appropriate buffer layer architecture, and fundamental control of the biaxial alignment of the initial buffer layer or underlying metallic substrate. A Defense Production Act, Title III program is being put in place to establishing long length manufacturing of this conductor at two contractor locations [8,9]. Development will continue on the basic YBCO conductor to exceed $100 \mathrm{~m}$ lengths and incorporate electrical stabilization of the wire.

With these advances and the establishment of reel-to-reel processing, new research should address the further enhancement of the performance of the YBCO coated conductors allowing additional reductions in size and weight of already compact power systems. Improvements of HTS conductor must focus on maintaining high current densities in fields of a few tesla while simultaneously minimizing low ac losses. Recent research activities at the Air Force Research Laboratory's Propulsion Directorate located at WrightPatterson AFB, OH are doing just that. The following sections discuss these conductor enhancements using flux pinning and ac loss design techniques.

\section{A. Flux Pinning}

In general, superconductors exhibit zero resistance when operating at temperatures below their maximum critical transition temperature, $T_{c}$. In the superconducting state, magnetic flux is expelled from the material which is commonly known as the Meissner effect. Exceptions to this, result in separation of the superconducting materials into two broad categories, types I and II. The type I materials are generally the pure metallic elements. Type II materials tend to be alloys and ceramics and are characterized by their ability to retain superconducting attributes in the presence of higher applied magnetic fields. The difference is that type II materials tolerate some degree of applied magnetic flux intrusion into their interiors without completely destroying the superconducting state.

In type II superconductors, fluxons of magnetic field begin to penetrate the material as either the applied magnetic field and/or temperature increases. This results in a mixed state of superconductivity; the material within the flux tubes is in a normal state while the material surrounding the flux tubes remains in a superconducting state of zero resistance. Superconducting screening currents circulate around the small non-superconducting cores. Since the type II materials support this mixed state, they can operate at higher temperatures while maintaining large supercurrent densities.

In equilibrium conditions, the lower critical field, $\mathrm{H}_{\mathrm{c} 1}$ is defined as when the magnetic flux begins to penetrate the type II superconductor. This magnetic flux exists as a lattice of quantized line vortices or fluxons. Bulk superconductivity is destroyed when the normal state (non-superconducting) cores overlap at the upper critical field, $\mathrm{H}_{\mathrm{c} 2}$. In isotropic materials such as $\mathrm{Nb}-\mathrm{Ti}$ and $\mathrm{Nb} 3 \mathrm{Sn}$, vortex lines are continuous. This is 
not always the case for HTS, where the weak superconductivity of the blocking layers can at higher fields and temperatures produce a stack of weakly coupled "pancake" vortices whose circulating screening currents are primarily confined within the superconducting copper oxide planes $[12,13]$.

In any case, superconductors will carry bulk $\mathrm{J}_{\mathrm{c}}$ only if there is a macroscopic fluxon density gradient. This gradient can be sustained by pinning the vortices at microstructural defects. The goal of this flux pinning is to take advantage of this scenario by increasing the amount of magnetic flux the material can "pin," thus increasing its operational envelope. Increasing the temperature or applied field weakens the potential wells at which vortices are pinned. The critical current density, $J_{c}$, is the maximum $\mathrm{J}$ in the superconducting state and defined by the balance of the pinning and Lorentz forces. When $J$ exceeds $J_{c}$, a superconductor switches into a dissipative, vortex-flow state driven by the Lorentz force $[12,13]$.

Various attempts have been made to stabilize or pin the flux vortices. Introducing impurities or defects into the superconducting material is a known way to provide flux pinning [14]. Such pinning sites can be holes, nanotubes, particles, grain boundaries or other microstructural defects intentionally (or unintentionally) introduced into the superconducting material. Flux pinning is a key factor in the development of superconducting generators-allowing the conductor to operate in a magnetic field of several tesla while preserving a high current density.

One method discovered in recent work by Haugan et al. is that of island growth of $\mathrm{Y}_{2} \mathrm{BaCuO}_{5}$ (Y211) nanoparticles, a nonsuperconducting phase of $\mathrm{YBCO}$, in a composite multilayer structure with $\mathrm{YBa}_{2} \mathrm{Cu}_{3} \mathrm{O}_{7-\mathrm{x}}(\mathrm{Y} 123)$, the superconducting phase of YBCO [15]. The Y211 particulate inclusion was accomplished by growing the Y123 layer and Y211 nanoparticles by multiple consecutive depositions with pulsed laser ablation of the respective targets on $\mathrm{LaAlO}_{3}$ substrates. Island growth of Y211 was caused by lattice mismatching with the Y123 achieving a surface particle density on the order of $10^{11} \mathrm{~cm}^{-2}$ with typical particle size of $\sim 15 \mathrm{~nm}$ diameter and $\sim 4$ $\mathrm{nm}$ height, see Figure 1. Composite multilayer structures of (Y211 particles / Y123 layers) $\mathrm{x} N$ were grown with each bilayer being several nanometers thick. The Y123 phase maintained excellent epitaxy and in-plane orientation even with the Y211 particles. The critical current densities increased 3-5 times at applied fields of $1.6 \mathrm{~T}, 30-77 \mathrm{~K}$ compared to plain Y123 films. This area particle density has been shown in the LTS NbTi and now in YBCO thin films with this work.

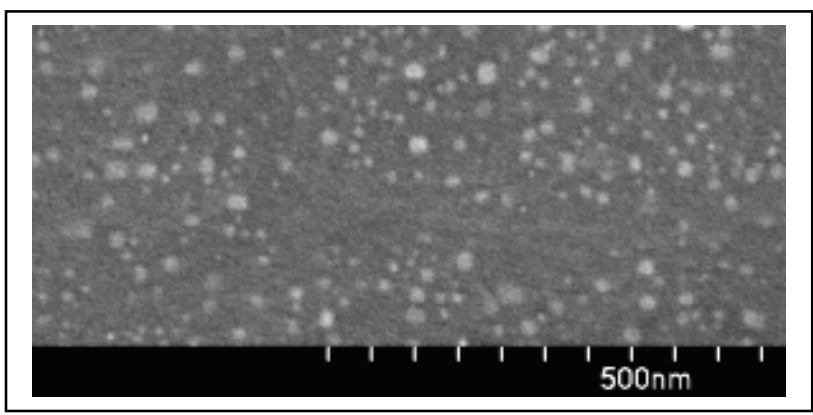

Figure 1. Y211 particles embedded on the YBCO layer.

\section{B. AC Losses}

Minimizing the ac losses in YBCO is critical to the development of high power, airborne generators, especially due to the high frequencies of operation (100's Hz) [16]. Losses in the YBCO coated conductor mainly result from externally applied alternating magnetic fields inducing hysteretic, ferromagnetic, eddy current, and coupling current losses. Losses may also be incurred from self-field transport currents. Reduction of ac losses in superconductors has been demonstrated before. Ultrafine filaments of $\mathrm{NbTi}$ were manufactured and built into a resistive $\mathrm{CuNi}$ matrix that minimized the ac loss in the fully penetrated field in the active length of a superconducting armature winding $[17,18]$. Creating multifilamentary YBCO is more difficult due to its wide, flat tape geometry. However, the tape can be subdivided and twisted, resulting in individual filaments of YBCO decoupled through the twist. This design parallels that of traditional LTS wire made of bundled individual wires [18].

Other issues include stability and quench protection. Stability is necessary for all conductors to ensure the HTS conductor remains in the superconducting state for the given application. Stability can be achieved by the inclusion of a conductive layer, e.g. $\mathrm{Cu}$ or $\mathrm{Ag}$, in direct contact with the YBCO. The amount of added stabilizer will vary with application, as well as the geometry of its inclusion. Quench protection is also needed to protect the conductor and device from any irreversible damage as a result of a quench. Quench protection is typically device dependent, even though various standard generic schemes are available.

The Air Force Research Laboratory demonstrated the reduction of hysteretic losses by subdividing the YBCO into thin filaments [19], see Figure 2. Magnetization vs. applied field measurements (M-H loops) were taken on short samples of YBCO films which were divided into narrow filaments. The YBCO was deposited using pulsed laser deposition onto singlecrystal $\mathrm{LaAlO}_{3}$ substrates. The thin films were patterned into linear striations by removing strips of the superconductor with a YAG laser. The resulting striated filamentary structure serves to reduce the effective width of the YBCO films and hence the hysteresis loss in the HTS samples. The magnetization measurements were taken over the temperature range of $4.2-$ $77 \mathrm{~K}$ in applied fields of $0-17 \mathrm{kOe}$ using a vibrating sample magnetometer. The measured hysteresis losses show a highly linear relationship between superconductor filament width and hysteresis loss as anticipated.

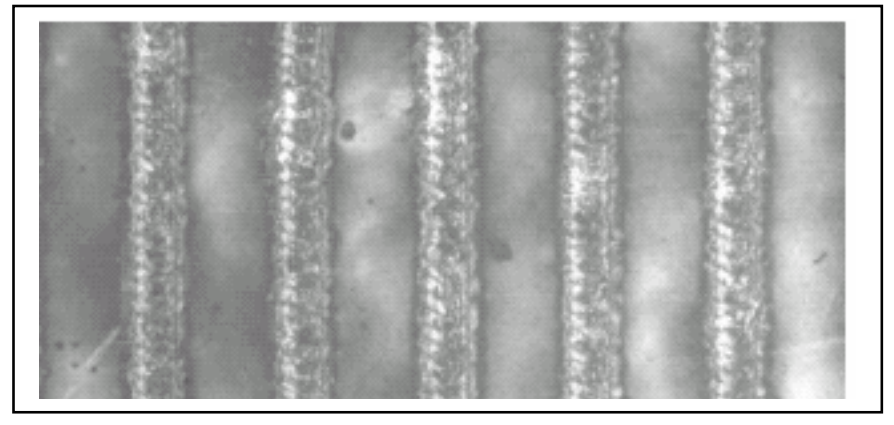

Figure 2. Striated YBCO. 
With successful development of an ac tolerant YBCO coated conductor, it can not only be used to make the coil windings in HTS generators, but potentially be used in HTS stator windings as well. As such, long lengths of the ac conductor with high current transport are required to achieve this goal in rotating machinery, and for use in power transformers. A multifilamentary YBCO tape may be the means of achieving this, and any potential breakage of the fine filaments in the YBCO can be mitigated by requiring current sharing between filaments. It must be such that the decrease in hysteretic and coupling losses derived from striation of the YBCO film is not completely lost by employing means which allow for some level of current sharing.

Current sharing might be useful by assisting in stability and quench protection. If a very small localized instability occurs within a filament, this filament can current share with all remaining filaments, thus improving the effective stability. On the other hand, the inevitable defects or variations in $\mathrm{J}_{\mathrm{c}}$ occurring within various regions of the conductor, made evident by the filamentarization, can be made less critical to the conductor performance by the ability to current share around these defects. These defects may arise either during the initial fabrication process, during the filamentarization process, or during subsequent handling.

Most superconducting materials are contained within metallic "carriers" and in practical cases metallic regions separate the filaments of multifilamentary conductors. In this case, the hysteretic losses are reduced by the filamentarization, while the metallic connection between the filaments allows current sharing within the conductor. This situation could also be induced for YBCO conductors, however, it is well known that this leads to a certain amount of coupling eddy current loss. This kind of loss is proportional to the interfilamentary conductivity, and thus gets worse as current sharing gets better. The coupling eddy current losses can be sufficient to nullify the effects of the filametarization. In high frequency applications, the filament recoupling is increased with increasing frequency. Thus, it difficult to use normal metal conductivity to enable current sharing in a multifilamentary strand intended for high frequency (100's Hz) applications. Superconducting currents, on the other hand, are frequency independent. Thus, it is possible to conceive of a low level HTS connection which will enable frequency independent current sharing, while keeping ac loss low. If done, it may allow fabrication of filamented YBCO conductors with low loss and maximal transport properties.

\section{CONCLUSION}

Future military systems will depend on high electrical power input that can range up to the megawatt level. These systems include electro-magnetic launch applications and electrically driven DEW. The power subsystems required for these applications must often be packaged using limited space and within strict weight limits on either ground-mobile or airborne platforms. Generators made of HTS will enable megawatt-class airborne or ground mobile power systems that are lightweight and compact. Key to these generators are the HTS conductors recently made available and advances in cryogenic refrigeration systems. HTS YBCO has demonstrated the best performance and is capable of meeting requirements of a superconducting power system. YBCO conductor can be further enhanced by better flux pinning methods and development of an ac tolerant architecture, which recent research has shown to be possible. These enhancements will lead to unprecedented power densities in generators.

\section{ACKNOWLEDGMENT}

We thank T. Haugan and C. Oberly for their assistance.

\section{REFERENCES}

[1] C.E. Oberly, "Air Force applications of lightweight superconducting machinery,” IEEE Trans. on Magnetics, Vol. 13, pp. 260-268, 1977.

[2] C.E. Oberly and J.C. Ho, "The origin and future of composite aluminum conductors," IEEE Trans. on Magnetics, Vol. 27, pp. 458-463, 1991.

[3] P.W. Eckels, "Cryogenic generator cooling," Adv. Cryo. Eng., Vol. 35B, pp. 1639-1646, 1990.

[4] A.J. Rodenbush and S.J. Young, "Performance of high temperature superconducting coils for implementation into megawatt class generators," IEE Trans. on Appl. Superconductivity, Vol. 9, pp. 12331236, 1999.

[5] Press Release, "AMSC successfully completes no-load factory tests-Proprietary 5 megawatt motor on target for delivery to U.S. Navy in July 2003," American Superconductor Corporation, Westborough, MA, March 25, 2003.

[6] C.E. Oberly, G. Kozlowski, C.E. Gooden, R.X. Lenard, A.K. Sarkar, I. Maartense, and J.C. Ho, "Principles of application of high temperature superconductors to electromagnetic launch technology," IEEE Trans. on Magnetics, Vol. 27, pp. 509-514, 1991.

[7] I.R. McNab, "Pulsed power for electric guns," IEEE Trans. on Magnetics, Vol. 33, pp. 453-460, 1997.

[8] American Superconductor Corporation, http://www.amsuper.com

[9] SuperPower, Inc., http://www.igc.com/superpower

[10] L.B. Penswick and B.P. Hoden, "Development of a light weight linear drive cryocooler for cryogenically cooled solid state laser systems," Cryocoolers, Vol. 10, pp.77-85, 1999 [Proceedings of the $10^{\text {th }}$ International Cryocooler Conference, May 26-28, 1998].

[11] J. Ekin, N. Cheggour, C. Clickner, M. Arecht, A. Adare, "Electromechnaical studies for coated-conductor development," presented at DOE Annual Peer Review - Superconductivity Program for Electric Systems, July 23-25, 2003.

[12] D. Larbalestier, A. Gurevich, D.M. Feldmann, A. Polyanskii, "High $T_{c}$ superconducting materials for electric power applications," Nature, Vol. 414, pp. 368-377, 2001.

[13] G. Blatter, M.V. Feigel'man, V. B. Geshkenbein, A.I. Larkin, V.M. Vinokur, "Vortices in high-temperature superconductors, Rev. Modern Phys., Vol. 66, pp. 1125-1364, 1994.

[14] T. Matsushita, "Flux pinning in superconducting 123 materials," Supercond. Sci. Technol., Vol. 13, pp. 730-737, 2000.

[15] T.J. Haugan, P.N. Barnes, I. Maartense, E.J. Lee, M. Sumption, and C.B. Cobb, "Island growth of $\mathrm{Y}_{2} \mathrm{BaCuO}_{5}$ nanoparticles in $\left(211_{\sim 1.5 \mathrm{~nm}} /\right.$ $\left.123_{\sim 0 \mathrm{~nm}}\right) \mathrm{XN}$ composite multilayer structures to enhance flux pinning of $\mathrm{YBa}_{2} \mathrm{Cu}_{3} \mathrm{O}_{7-\mathrm{d}}$ films," J. Mater. Res., Vol. 18, pp. 2618-2623, 2003.

[16] C.E. Oberly, G.L. Rhoads, P.N. Barnes, L. Long, D.J. Scott, and W.J. Carr Jr., "The importance of interfilamentary barrier resistance in YBCO coated conductor to minimize ac losses, Adv. in Cryogenic Eng., Vol. 48B, pp. 621-630, 2002.

[17] J. R. Cave, A. Fervrier, and T. Verhahaege, "Reduction of ac losses in ultra-fine multifilamentary NbTi wires," IEEE Trans. on Magnetics, Vol. 25, pp. 1945-1948, 1989.

[18] C.E. Oberly, L. Long, G.L. Rhoads, W.J. Carr, "Loss minimization in Y$\mathrm{Ba}-\mathrm{Cu}-\mathrm{O}$ coated conductors with fully penetrated ac magnetic field," Cryogenics, Vol. 41, pp. 117-124, 2001.

[19] C.B. Cobb, P.N. Barnes, T.J. Haugan, J.C. Tolliver, E.J. Lee, M.D. Sumption, E.W. Collings, C.E. Oberly, "Hysteretic loss reduction in striated YBCO," Physica C, Vol. 382, pp. 52-56, 2002. 